\title{
Japan
}

\section{"Antibacterial Composition"}

Patent Act, Sec. 36(6)(i) - Well-Being Biochemical Co., Ltd.

\section{v. The Commissioner of the Patent Office}

\section{Decision of the Intellectual Property High Court}

26 December 2011

(C) Max Planck Institute for Intellectual Property and Competition Law, Munich 2013

Experimental results submitted after the filing date of the patent cannot be taken into account where they confirm an effect that has not been described in the patent application.

The description of a patented invention about an "antibacterial, antiviral and antimycotic composition", in which one of the components specified by the claim should be selected among several metallic ion compounds, provided concrete data only for the composition comprising a certain compound (copper) in the detailed explanation of the invention. Referring to this disclosure, a person skilled in the art could determine whether the expected effect could be achieved over the entire range of the compounds specified by the claim. As such, the patent lacked sufficient disclosure for the whole range claimed. The experimental results supplied only after the patent application date with respect to matters not described in the original specification could not be taken into account to the extent that they referred to the experimental data for compositions comprising other compounds.

Summarised by Hisayoshi Yokoyama. 